\title{
QUEEN'S
UNIVERSITY
BELFAST
}

\section{3-D Position and Velocity Estimation in 5G mmWave CRAN with Lens Antenna Arrays}

Yang, J., Jin, S., Han, Y., Matthaiou, M., \& Zhu, Y. (2019). 3-D Position and Velocity Estimation in 5 G mmWave CRAN with Lens Antenna Arrays. In 90th IEEE Vehicular Technology Conference (VTC): Proceedings (Vehicular Technology Conference (VTC): Proceedings). Institute of Electrical and Electronics Engineers Inc.. https://doi.org/10.1109/VTCFall.2019.8891333

Published in:

90th IEEE Vehicular Technology Conference (VTC): Proceedings

Document Version:

Peer reviewed version

Queen's University Belfast - Research Portal:

Link to publication record in Queen's University Belfast Research Portal

Publisher rights

(c) 2019 IEEE.

3-D Position and Velocity Estimation in $5 \mathrm{G}$ mmWave CRAN with Lens Antenna Arrays

\section{General rights}

Copyright for the publications made accessible via the Queen's University Belfast Research Portal is retained by the author(s) and / or other copyright owners and it is a condition of accessing these publications that users recognise and abide by the legal requirements associated with these rights.

Take down policy

The Research Portal is Queen's institutional repository that provides access to Queen's research output. Every effort has been made to ensure that content in the Research Portal does not infringe any person's rights, or applicable UK laws. If you discover content in the Research Portal that you believe breaches copyright or violates any law, please contact openaccess@qub.ac.uk. 


\title{
3-D Position and Velocity Estimation in 5G mmWave CRAN with Lens Antenna Arrays
}

\author{
Jie Yang ${ }^{1}$, Shi Jin ${ }^{1}$, Yu Han ${ }^{1}$, Michail Matthaiou ${ }^{2}$, and Yongxu Zhu ${ }^{3}$ \\ ${ }^{1}$ National Mobile Communications Research Laboratory, Southeast University, Nanjing, China \\ ${ }^{2}$ Institute of Electronics, Communications and Information Technology (ECIT), Queen's University Belfast, Belfast, U.K. \\ ${ }^{3}$ Wolfson School of Mechanical, Electrical and Manufacturing Engineering, Loughborough University, Leicestershire, U.K. \\ E-mail: ${ }^{1}\left\{\right.$ yangjie, jinshi, hanyu\}@ seu.edu.cn, ${ }^{2}$ m.matthaiou@qub.ac.uk, ${ }^{3}$ y.zhu4@lboro.ac.uk
}

\begin{abstract}
G millimeter-wave (mmWave) cloud radio access network (CRAN) provides new opportunities for accurate multilateration: large bandwidth, large antenna arrays, and increased densities of base stations allow for unparalleled delay and angle resolution. However, combining localization into communications and designing joint position and velocity estimation algorithms are challenging. This paper considers the joint estimation in three-dimensional (3-D) lens antenna array based mmWave CRAN architecture. We embed multilateration into communications and explain its benefits to the initial access and beam training stages. We propose a closed-form solution for the joint estimation problem by forming the pseudo-linear matrix representation and designing the weighted least squares estimator with hybrid measurements. The proposed method is proven asymptotically unbiased and confirmed by simulations to achieve the Cramer-Rao lower bound and attain the sub-decimeter level accuracy.
\end{abstract}

Index Terms-CRAN, hybrid measurements, lens antenna array, localization, mmWave.

\section{INTRODUCTION}

Communication at millimeter-wave (mmWave) frequencies is viewed as a promising technique for $5 \mathrm{G}$ and future wireless communications [1]. Since the mmWave band can offer more available spectrum and larger bandwidth than presently used sub-6 GHz bands, a higher resolution of the time delay (TD), time difference of arrival (TDoA) and frequency difference of arrival (FDoA) can be obtained. In addition, the penetration loss at mmWave bands is inherently large [2], [3], hence, the gap of received power between line-of-sight (LoS) path and non-line-of-sight (NLoS) path is exacerbated, which makes it easier to eliminate the NLoS interference [3]. To compensate for the severe penetration loss and the increased path-loss, large antenna arrays and highly directional transmission should be combined [4], which facilitates the acquisition of angle of arrival (AoA) and angle of departure (AoD). Moreover, cloud radio access network (CRAN) can also enhance mmWave communications by improving the network coverage [5]. Therefore, mmWave CRAN can offer accurate localization, in return, the location information can improve the scalability, latency, and robustness of the future networks [6].

CRAN provides a cost-effective way of achieving network densification, in which distributed low-complexity remote radio heads (RRHs) are deployed close to the users and coordinated by a central unit (CU) for joint processing. More powerful processing ability of $\mathrm{CU}$ than user equipment (UE) makes it possible to implement multilateration in uplink. Works in [7], [8] were designed for immobile targets and used in radar systems. Study in [9] analyzed the localization performance by accounting for the distortion caused by fronthaul links quantization, however, the work was limited to the twodimensional (2-D) scenario with coarse positioning accuracy. The works of [10], [11] estimated the location and velocity of the moving source in radar systems. The algorithm in [10] estimated the position and velocity separately, and the research in [11] was limited to 2-D localization. The work in [12] studied three-dimensional (3-D) downlink positioning with single reference station, which requires both AoA and AoD measurements (obtained after beam training) and abundant single-bounce multipath components.

In this paper, we focus on the joint position and velocity estimation problem of the moving UE. We adopt mmWave CRAN architecture [5], and leverage advanced 3-D lens antenna arrays at the RRHs. Lens can operate at very short pulse length and receive signals with different AoAs simultaneously without beam scanning [4], which can accelerate the uplink localization process. Moreover, lens antenna arrays are effective in exploiting the sparsity of the mmWave channel. Since lens has energy focusing property, signals with different AoAs are brought to different antenna elements on the focal surface, and only few of the antenna elements receive significant power, which can simplify the signal processing [13]. This paper embeds multilateration into mmWave communications and proposes a closed-form solution for the joint estimation problem by using TDoA, FDoA, and AoA hybrid measurements, without the knowledge of the AoD and clock biases. The proposed method can obtain the position and velocity of the UE simultaneously.

\section{SYSTEM MODEL}

We study the localization (position and velocity estimation of the moving UE) in mmWave CRAN with $N$ multi-antenna RRHs [5], as shown in Fig. 1, where each RRH is equipped with $J=3$ 3-D lens antenna arrays, and each lens antenna array covers $120^{\circ}$ and has $M$ antenna elements. Each RRH is connected to the $\mathrm{CU}$ via an individual fronthaul link of finite capacity. There are $K$ single antenna UEs in CRAN1, where

\footnotetext{
${ }^{1}$ The total transmission power limit of the UE can be compensated in the future study by utilizing multi-antennas and beam scanning in the UE side.
} 


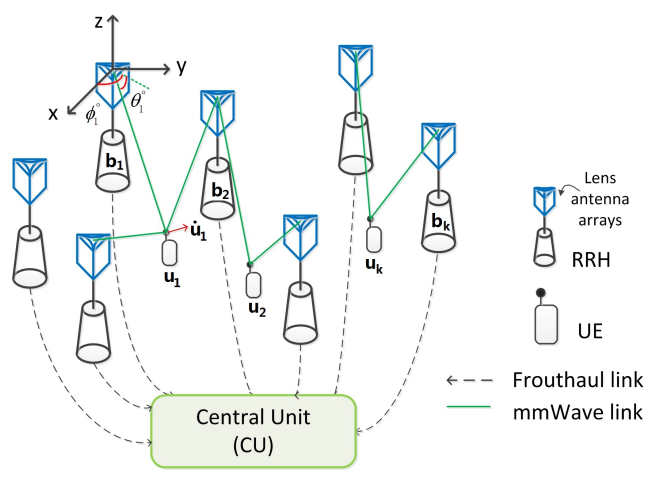

Fig. 1. $\quad N$ RRHs locate at $\mathbf{b}_{n}=\left[x_{b, n}, y_{b, n}, z_{b, n}\right]^{T}$, for $n=1,2, \ldots, N$ $K$ UEs locate at $\mathbf{u}_{k}=\left[x_{k}, y_{k}, z_{k}\right]^{T}$ with velocity $\dot{\mathbf{u}}_{k}=\left[\dot{x}_{k}, \dot{y}_{k}, \dot{z}_{k}\right]^{T}$, for $k=1,2, \cdots, K$. The AoA pair of the LoS path between RRH 1 and UE 1 is $\left(\phi_{1}, \theta_{1}\right)$.

the number of UEs is no greater than $J N$. Depending on a UE's location, its signals are incident on at most one lens antenna array of each RRH. We consider a 3-D space $\mathbb{R}^{3}=$ $\left\{[x, y, z]^{T}: x, y, z \in \mathbb{R}\right\}$ with $N$ known RRHs located at $\mathbf{b}_{n}=\left[x_{b, n}, y_{b, n}, z_{b, n}\right]^{T}, n=1,2, \cdots, N$, where $(\cdot)^{T}$ denotes the transpose. The geometry between the RRHs and the UEs is shown in Fig. 1] We assume that the unknown position and velocity of the $k$-th UE is represented by $\mathbf{u}_{k}=\left[x_{k}, y_{k}, z_{k}\right]^{T}$ and $\dot{\mathbf{u}}_{k}=\left[\dot{x}_{k}, \dot{y}_{k}, \dot{z}_{k}\right]^{T}, k=1,2, \cdots, K$, respectively.

\section{A. Channel Model}

The UEs transmit over a mmWave wideband frenquencyselective channel. The channel between UE $k$ and RRH $n$ has $C_{n, k}$ clusters containing $L_{n, k, c}$ paths each. Since the mmWave channel is sparse, we assume that $\sum_{c} C_{n, k} L_{n, k, c} \leq J Q$, where $Q$ is the number of radio frequency (RF) chains for each lens antenna array. Hence, the channel coefficient vector $\mathbf{h}_{n, k}(t) \in \mathbb{C}^{J M \times 1}$ at time $t$ between UE $k$ and RRH $n$ can be expressed using the clustered channel model [14] as

$$
\mathbf{h}_{n, k}(t)=\sum_{c=0}^{C_{n, k}} \sum_{l=0}^{L_{n, k, c}} \alpha_{n, k, c, l} \delta\left(t-\tau_{n, k, c, l}\right) \mathbf{a}\left(\phi_{n, k, c, l}, \theta_{n, k, c, l}\right)
$$

where $\alpha_{n, k, c, l}, \tau_{n, k, c, l}, \phi_{n, k, c, l}, \theta_{n, k, c, l}$ denote the complex gain, delay, azimuth and elevation AoA, for the $l$-th path of the $c$-th cluster, respectively. $\mathbf{a}(\cdot)$ is the array response vector of the lens antenna array at RRH.

We consider that each rectangular lens equipped at RRH has dimension of $\tilde{D}_{y} \times \tilde{D}_{z}$ normalized by the wavelength along the $y$ - and $z$-axes, respectively. The 3-D lens has $M$ antenna elements placed on the focal surface, as shown in Fig. 2 Let each antenna element be indexed by a pair of indexes $\left(m_{a}, m_{e}\right)$, where $m_{a} \in\left[1, M_{a}\right]$ and $m_{e} \in\left[1, M_{e}\right]$ denote azimuth and elevation index, respectively, with $M=M_{a} \times M_{e}$. According to [4], for a uniform plane wave incident at azimuth and elevation angles $(\phi, \theta)$, reshaping $M \times 1$ array response vector a $(\phi, \theta)$ into a $M_{a} \times M_{e}$ matrix, the $\left(m_{a}, m_{e}\right)$-th element in the matrix is given by

$$
a_{m_{a}, m_{e}}(\phi, \theta) \propto \operatorname{sinc}\left(m_{a}-\tilde{D}_{y} \sin \phi\right) \operatorname{sinc}\left(m_{e}-\tilde{D}_{z} \cos \phi \sin \theta\right) .
$$

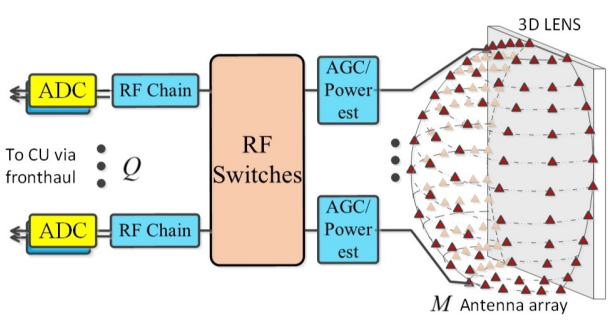

Fig. 2. Architecture of RRH with lens antenna array.

From (2), it is obvious that the antenna element $\left(m_{a}, m_{e}\right)$, which has the strongest received power, is corresponding to AoA pair $(\phi, \theta)$. Since the RRHs are typically low-cost nodes with limited processing ability and RF chains, and each antenna element is equipped with the automatic gain control (AGC) circuitry, we assume that RRHs are able to perform antenna selection based on the estimated received power levels at each antenna element. After antenna selection, the indexes of selected antennna elements have one-to-one correspondence with AoAs.

\section{B. Transmission Model}

Positioning can be embeded in either initial access stage or data transmission stage without additional overhead. Take initial access [15] for example: each RRH periodically transmits synchronization signals to UEs, after detecting the synchronization signals and decoding the broadcast messages, the UE randomly selects one of a small number (in LTE, there are up to 64) of waveforms, called random access (RA) preambles, and transmits it in one of the RA slots. Hence, we can assume that the uplink interference from other UEs does not need to be considered due to the near orthogonality in waveform and time. Therefore, we take one UE to simplify expressions.

The UE omni-directionally sends a signal $\sqrt{E_{s}} s(t)$, where $E_{s}$ is the transmitted energy, and $\mathbb{E}\left\{s(t) s^{H}(t)\right\}=1$, where $(\cdot)^{H}$ denotes the conjugate transpose. The received signal $\mathbf{r}_{n}(t) \in \mathbb{C}^{J Q \times 1}$ at $n$-th RRH is given by

$\mathbf{r}_{n}(t)=\mathbf{W}_{s} \sum_{c=0}^{C_{n}} \sum_{l=0}^{L_{n, c}} \alpha_{n, c, l} \sqrt{E_{s}} s\left(t-\tau_{n, c, l}\right) \mathbf{a}\left(\phi_{n, c, l}, \theta_{n, c, l}\right)+\mathbf{n}(t)$,

where $\mathbf{W}_{s} \in \mathbb{R}^{J Q \times J M}$ is $\mathrm{RF}$ switch network designed according to the AGC feedbacks, with elements of 0 and 1 (only one 1 in each row). $\mathbf{n}(t) \in \mathbb{C}^{J Q \times 1}$ is zero-mean white Gaussian noise with known power spectrum density. In this paper, we focus on the proposed localization algorithm itself and ignore the impact of quantization in the fronthaul links, which will be considered in the future work. Then, $J Q$ distinct paths with known AoAs in RRH $n$ are transmitted to the CU, for $n=1, \cdots, N$. The LoS path can be identified easily for mmWave, since it is much stronger than the NLoS path. We assume that the threshold should be selected so that the $\mathrm{CU}$ can generally select $N_{s} \geq 2 \mathrm{LoS}$ paths from $N$ RRHs in order to ensure good localization accuracy. For simplicity, we denote the delay and the AoA pairs of the $n$-th $\operatorname{LoS}$ path as $\tau_{n}$ and 
$\left(\phi_{n}, \theta_{n}\right)$, for $n=1, \cdots, N_{s}$. After the baseband processing, the delay $\tau_{n}$ for $n$-th LoS path can also be determined ${ }^{2}$ Finally, the CU obtains the channel parameters $\left(\phi_{n}, \theta_{n}, \tau_{n}\right)$ of $n$-th $\operatorname{LoS}$ path for $n=1, \cdots, N_{s}$, which are necessary to locate the UE.

\section{Proposed Solution}

In this section, we propose an effective localization method, which can obtain the unknown position $\mathbf{u}^{\circ}=\left[x^{\circ}, y^{\circ}, z^{\circ}\right]^{T}$ and velocity $\dot{\mathbf{u}}^{\circ}=\left[\dot{x}^{\circ}, \dot{y}^{\circ}, \dot{z}^{\circ}\right]^{T}$ of the UE simultaneously $]^{3}$

\section{A. Relation Between Channel and Location Parameters}

We can obtain delays $\tau_{n}$ and AoA pairs $\left(\phi_{n}, \theta_{n}\right)$ for $n$ th LoS path, as described in Section II-B. These channel parameters will be mapped to location parameters in the following.

a) TDoAs: For the $n$-th LoS path, the TD is given by

$$
\tau_{n}=\left\|\mathbf{u}^{\circ}-\mathbf{b}_{n}\right\| / v_{c}+\omega
$$

where $\|\cdot\|$ denotes the 2-norm, $\omega$ is the unknown clock bias, and $v_{c}$ is the speed of light. The TDoA of a signal received by the RRH $n$ and 1 is given by

$$
\Delta \tau_{n}=\tau_{n}-\tau_{1},
$$

where the unknown $\omega$ is cancelled. Let

$$
r_{n}^{\circ}=\left\|\mathbf{u}^{\circ}-\mathbf{b}_{n}\right\|=\sqrt{\left(\mathbf{u}^{\circ}-\mathbf{b}_{n}\right)^{T}\left(\mathbf{u}^{\circ}-\mathbf{b}_{n}\right)},
$$

and

$$
r_{n 1}^{\circ}=r_{n}^{\circ}-r_{1}^{\circ},
$$

where $r_{n 1}^{\circ}$ is the TDoA related parameters used in the proposed algorithm, derived from the TDoA by multiplying $v_{c}$.

b) FDoAs: The FDoA is the change rate of the TDoA with time. The time derivative of (6) leads to

$$
\dot{r}_{n}^{\circ}=\dot{\mathbf{u}}^{\circ T}\left(\mathbf{u}^{\circ}-\mathbf{b}_{n}\right) / r_{n}^{\circ} .
$$

We define that

$$
\dot{r}_{n 1}^{\circ}=\dot{r}_{n}^{\circ}-\dot{r}_{1}^{\circ},
$$

where $\dot{r}_{n 1}^{\circ}$ is the FDoA related parameters, derived from the FDoA by multiplying $v_{c}$, between the RRH $n$ and 1 . The FDoA is used to estimate the velocity of the UE, with a short time accumulation, the $\mathrm{CU}$ can obtain the change rate of the TDoA.

c) AoAs: For the LoS path, there hold

$$
\phi_{n}^{\circ}=\arctan \frac{y^{\circ}-y_{b, n}}{x^{\circ}-x_{b, n}}, \quad \theta_{n}^{\circ}=\arcsin \frac{z^{\circ}-z_{b, n}}{\left\|\mathbf{u}^{\circ}-\mathbf{b}_{n}\right\|} .
$$

The hybrid measurements (TDoA, FDoA, AoA) will be utilized to estimate the position and velocity of the UE. Since the relationships (6)-(10) are nonlinear and non-convex functions of $\mathbf{u}^{\circ}$ and $\dot{\mathbf{u}}^{\circ}$, solving the locating problem is

\footnotetext{
${ }^{2}$ The time delay is estimated by detecting the first peak of the correlation between the received signal and the transmitted reference signal, e.g. PRS.

${ }^{3}$ In the following paper, the notation $a^{\circ}$ represents the true value of the estimated parameter $a$.
}

not a trivial task. In the following sections, a WLS-based positioning estimator will be proposed, and it will be proved to be asymptotically unbiased.

\section{B. Joint Estimation of Position and Velocity}

We firstly obtain the matrix representation of the joint position and velocity estimation model. Let

$$
\begin{aligned}
& \mathbf{a}_{n}^{\circ}=\left[\cos \theta_{n}^{\circ} \cos \phi_{n}^{\circ}, \cos \theta_{n}^{\circ} \sin \phi_{n}^{\circ}, \sin \theta_{n}^{\circ}\right]^{T}, \\
& \mathbf{c}_{n}^{\circ}=\left[-\sin \phi_{n}^{\circ}, \cos \phi_{n}^{\circ}, 0\right]^{T}, \\
& \mathbf{d}_{n}^{\circ}=\left[-\sin \theta_{n}^{\circ} \cos \phi_{n}^{\circ},-\sin \theta_{n}^{\circ} \sin \phi_{n}^{\circ}, \cos \theta_{n}^{\circ}\right]^{T},
\end{aligned}
$$

for $n=1, \cdots, N_{s}$. By applying the nonlinear transformation to (6)-(9) and utilizing the angular relationships in (11), we obtain the noise-free matrix representation of the joint position and velocity estimation model, given by

$$
\mathbf{h}=\mathbf{G} \mathbf{w}^{\circ},
$$

where $\mathbf{w}^{\circ}=\left[\mathbf{u}^{\circ T}, \dot{\mathbf{u}}^{\circ T}\right]^{T}$ is the vector of unknown position and velocity of the UE, $\mathbf{h}=\left[\mathbf{q}_{2}^{T}, \ldots, \mathbf{q}_{N_{s}}^{T}, \mathbf{h}_{1}^{T}, \ldots, \mathbf{h}_{N_{s}}^{T}\right]^{T}$, $\mathbf{G}=\left[\mathbf{P}_{2}^{T}, \ldots, \mathbf{P}_{N_{s}}^{T}, \mathbf{G}_{1}^{T}, \ldots, \mathbf{G}_{N_{s}}^{T}\right]^{T}$, and

$$
\begin{aligned}
& \mathbf{q}_{n}=\left(\begin{array}{c}
\left(r_{n 1}^{\circ}\right)^{2}-2 r_{n 1}^{\circ} \mathbf{a}_{1}^{\circ T} \mathbf{b}_{1}-\mathbf{b}_{n}^{T} \mathbf{b}_{n}+\mathbf{b}_{1}^{T} \mathbf{b}_{1} \\
\dot{r}_{n 1}^{\circ} r_{n 1}^{\circ}-\dot{r}_{n 1}^{\circ} \mathbf{a}_{1}^{\circ T} \mathbf{b}_{1}
\end{array}\right), \quad \mathbf{h}_{n}=\left(\begin{array}{c}
\mathbf{c}_{n}^{\circ T} \mathbf{b}_{n} \\
\mathbf{d}_{n}^{\circ T} \mathbf{b}_{n}
\end{array}\right), \\
& \mathbf{P}_{n}=\left(\begin{array}{cc}
2\left[\left(\mathbf{b}_{1}-\mathbf{b}_{n}\right)^{T}-r_{n 1}^{\circ} \mathbf{a}_{1}^{\circ T}\right] & \mathbf{0} \\
-\dot{r}_{n 1}^{\circ} \mathbf{a}_{1}^{\circ T} & \left(\mathbf{b}_{1}-\mathbf{b}_{n}\right)^{\frac{T}{T}} r_{n 1}^{\circ} \mathbf{a}_{1}^{\circ T}
\end{array}\right), \mathbf{G}_{n}=\left(\begin{array}{cc}
\mathbf{c}_{n}^{\circ T} & \mathbf{0} \\
\mathbf{d}_{n}^{\circ T} & \mathbf{0}
\end{array}\right),
\end{aligned}
$$

and $\mathbf{0}$ is a $1 \times 3$ zero vector. The detailed derivations of (12) are listed in Appendix A.

Note that elements in vector $\mathbf{h}$ and matrix $\mathbf{G}$ in 12 are operations of the noise-free channel parameters. Let measured parameters with noise replace the true parameters in (12), namely, let $\left\{r_{i 1}=r_{i 1}^{\circ}+\Delta r_{i 1}, \dot{r}_{i 1}=\dot{r}_{i 1}^{\circ}+\Delta \dot{r}_{i 1}, \phi_{j}=\right.$ $\left.\phi_{j}^{\circ}+\Delta \phi_{j}, \theta_{j}=\theta_{j}^{\circ}+\Delta \theta_{j}\right\}$ replace $\left\{r_{i 1}^{\circ}, \dot{r}_{i 1}^{\circ}, \phi_{j}^{\circ}, \theta_{j}^{\circ}\right\}$ in $\mathbf{h}$ and $\mathbf{G}$, for $i=2, \cdots, N_{s}$ and $j=1, \cdots, N_{s}$, we get

$$
\tilde{\mathbf{h}}=\tilde{\mathbf{G}} \mathbf{w}^{\circ}+\mathbf{e},
$$

where $\tilde{\mathbf{h}}$ and $\tilde{\mathbf{G}}$ are measured counterparts, and $\mathbf{e}$ is the error vector. Equation (13) reflects the pseudo-linear relationship between the measured channel parameters and the unknown location parameters.

\section{WLS-based Joint Estimation Algorithm}

Relying on the proposed model in (13), we are able to obtain the weighted least squares (WLS) estimation of $\mathbf{w}^{\circ}$ according to [16]. Denote

$$
\begin{gathered}
\mathbf{m}=\left[r_{21}, \dot{r}_{21}, \ldots, r_{N_{s} 1}, \dot{r}_{N_{s} 1}, \phi_{1}, \theta_{1}, \cdots, \phi_{N_{s}}, \theta_{N_{s}}\right]^{T}, \\
\mathbf{m}^{\circ}=\left[r_{21}^{\circ}, \dot{r}_{21}^{\circ}, \ldots, r_{N_{s} 1}^{\circ}, \dot{r}_{N_{s} 1}^{\circ}, \phi_{1}^{\circ}, \theta_{1}^{\circ}, \ldots, \phi_{N_{s}}^{\circ}, \theta_{N_{s}}^{\circ}\right]^{T},
\end{gathered}
$$

and

$\Delta \mathbf{m}=\left[\Delta r_{21}, \Delta \dot{r}_{21} ; \cdots, \Delta r_{N_{s} 1}, \Delta \dot{r}_{N_{s} 1}, \Delta \phi_{1}, \Delta \theta_{1} ; \cdots, \Delta \phi_{N_{s}}, \Delta \theta_{N_{s}}\right]^{T}$,

then, we have

$$
\mathbf{m}=\mathbf{m}^{\circ}+\Delta \mathbf{m},
$$


where $\mathbf{m}$ is the vector of measured parameters, $\mathbf{m}^{\circ}$ is the vector of true parameters, and $\Delta \mathbf{m}$ is the vector of noise terms. We assume that $\Delta \mathbf{m}$ is a zero-mean Gaussian vector, whose variance matrix is $\mathbf{Q}$.

Proposition 1. The WLS estimation of $\mathbf{w}^{\circ}$ is given by

$$
\mathbf{W}=\left(\tilde{\mathbf{G}}^{T} \mathbf{W} \tilde{\mathbf{G}}\right)^{-1} \tilde{\mathbf{G}}^{T} \mathbf{W} \tilde{\mathbf{h}},
$$

where the positive definite weighting matrix $\mathbf{W}$ is taken to minimize the variance of $\mathrm{w}$. When the variance matrix $\mathbf{Q}$ is known, the weighting matrix can be deduced as follows:

$$
\mathbf{W}=\left(\mathbf{B Q B}^{T}\right)^{-1},
$$

where $\mathbf{B}$ (see (37) in Appendix B) is the coefficient matrix with $\mathbf{B} \Delta \mathbf{m}$ approximating the linear term of $\mathbf{e}$, namely, $\mathbf{e} \doteq$ $\mathbf{B} \Delta \mathbf{m}$, where $\doteq$ denotes "approximately equal". Hence, the WLS estimation is given by

$$
\mathbf{w}=\left(\tilde{\mathbf{G}}^{T}\left(\mathbf{B Q B} \mathbf{B}^{T}\right)^{-1} \tilde{\mathbf{G}}\right)^{-1} \tilde{\mathbf{G}}^{T}\left(\mathbf{B Q B} \mathbf{B}^{T}\right)^{-1} \tilde{\mathbf{h}} .
$$

Proof. See Appendix B.

Then, we evaluate the performance of the proposed estimator in 20, by analyzing the expectation of it. Asymptotic unbiasedness - the expectation of an estimator equals to the true value of the estimated parameters, which is a criterion for evaluating the validity of the estimator.

Lemma 1. The presented estimator $\mathbf{w}=\left(\tilde{\mathbf{G}}^{T} \mathbf{W} \tilde{\mathbf{G}}\right)^{-1} \tilde{\mathbf{G}}^{T} \mathbf{W} \tilde{\mathbf{h}}$ with $\mathbf{W}=\left(\mathbf{B Q B}^{T}\right)^{-1}$ is asymptotically unbiased, namely, $E\{\mathbf{w}\} \doteq \mathbf{w}^{\circ}$.

Proof. The true value $\mathbf{w}^{\circ}$ of $\mathbf{w}$ can be expressed by $\left(\tilde{\mathbf{G}}^{T} \mathbf{W} \tilde{\mathbf{G}}\right)^{-1}\left(\tilde{\mathbf{G}}^{T} \mathbf{W} \tilde{\mathbf{G}}\right) \mathbf{w}^{\circ}$. Then, from 18 , we yield

$$
\Delta \mathbf{w}=\mathbf{w}-\mathbf{w}^{\circ}=\left(\tilde{\mathbf{G}}^{T} \mathbf{W} \tilde{\mathbf{G}}\right)^{-1} \tilde{\mathbf{G}}^{T} \mathbf{W e} .
$$

Taking expectation in 21] indicates that

$$
E\{\Delta \mathbf{w}\}=E\{\mathbf{w}\}-\mathbf{w}^{\circ}=\left(\tilde{\mathbf{G}}^{T} \mathbf{W} \tilde{\mathbf{G}}\right)^{-1} \tilde{\mathbf{G}}^{T} \mathbf{W} E\{\mathbf{e}\} .
$$

As $E\{\mathbf{e}\} \doteq E\{\mathbf{B} \Delta \mathbf{m}\}=\mathbf{B} E\{\Delta \mathbf{m}\}=0$, we have $E\{\Delta \mathbf{w}\} \doteq 0$, therefore $E\{\mathbf{w}\} \doteq \mathbf{w}^{\circ}$.

Remark 1: We have proved that the proposed estimator is asymptotically unbiased, which means that the proposed algorithm will be more accurate as the number of measurements increases.

The proposed algorithm is summarized in Algorithm 1 . where $\mathbf{w}(i: j)$ represents the $i$-th to $j$-th entries in $\mathbf{w}$, and $T$ represents the total iterations. Note that the weighting matrix $\mathbf{W}$ in (19) is dependent on the unknown location $\mathbf{u}^{\circ}$ and velocity $\dot{\mathbf{u}}^{\circ}$ via matrix $\mathbf{B}$ defined in (37). Hence, in line 1 of Algorithm 1, we initialize $\mathbf{W}$ with $\mathbf{Q}^{-1}$. Then, in line 2 and 3 we obtain the coarse estimation w. Finally, by the update of $\mathbf{W}$ according to line $5-7$, we can produce a more accurate solution of $\mathbf{w}$ in line 8 .

Remark 2: 5G mmWave uplink localization can be executed during the initial access stage, as explained in Section II-B.

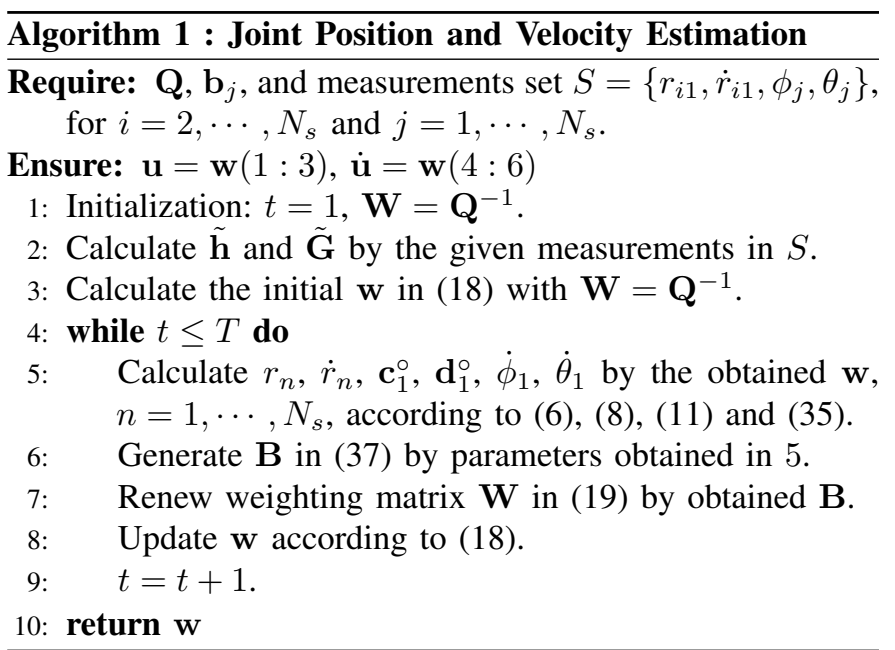

It can also be collaterally executed during the uplink channel estimation stage, with the estimated channel parameters, position and velocity information of the UE can be obtained. Then, the location information can be used to predict the state of the UE at the next epoch, which facilitates the initial access, beam training, and channel tracking process.

\section{NuMERICAL RESUlTS}

We consider a scenario with $N=6$ RRHs, located at $[-400,0,0]^{T},[400,0,0]^{T},[200,350,0]^{T},[-200,350,0]^{T}$, $[-200,-350,0]^{T},[200,-350,0]^{T}$ in meters, respectively. The UE is located at $\mathbf{u}^{\circ}=[300,-20,-100]^{T}(\mathrm{~m})$ with the velocity $\dot{\mathbf{u}}^{\circ}=[-9,7,5]^{T}(\mathrm{~m} / \mathrm{s})$. The CU select $N_{s}=2$ to 6 RRHs with LoS paths to locate the UE with the proposed localization method. To focus on the performance of the proposed algorithm, we do not take quantitative analysis of the measurement error caused by angular grid size of the lens and the fronthaul link quantization. For simplicity, we assume that the measurement variance $\mathbf{Q}$ indicates the impacts of all above factors leading to measurement error. The representation of $\mathbf{Q}$ is given by

$$
\mathbf{Q}=\operatorname{blkdiag}(\overbrace{\mathbf{Q}_{d}, \cdots, \mathbf{Q}_{d}}^{\left(N_{s}-1\right) \mathbf{Q}_{d}}, \overbrace{\mathbf{Q}_{a}, \cdots, \mathbf{Q}_{a}}^{N_{s} \mathbf{Q}_{a}}),
$$

where blkdiag is block diagonal with $\mathbf{Q}_{d}=\operatorname{diag}\left(\sigma_{d}^{2},\left(0.1 \sigma_{d}\right)^{2}\right)$ and $\mathbf{Q}_{a}=\operatorname{diag}\left(\sigma_{a}^{2}, \sigma_{a}^{2}\right)$, where $\sigma_{d}, 0.1 \sigma_{d}$, and $\sigma_{a}$ are the standard deviation for TDOA, FDoA, and AoA measurements, respectively. The iterations $T$ is set to 5 . The localization accuracy is assessed via the root-mean-square error $\operatorname{RMSE}(\mathbf{u})=\sqrt{\sum_{l=1}^{L}\left\|\mathbf{u}^{(l)}-\mathbf{u}^{\circ}\right\|^{2} / L}$, and $\operatorname{RMSE}(\dot{\mathbf{u}})=$ $\sqrt{\sum_{l=1}^{L}\left\|\dot{\mathbf{u}}^{(l)}-\dot{\mathbf{u}}^{\circ}\right\|^{2} / L}$, where $\mathbf{u}^{(l)}\left(\right.$ or $\left.\dot{\mathbf{u}}^{(l)}\right)$ is the estimation of $\mathbf{u}^{\circ}\left(\right.$ or $\left.\dot{\mathbf{u}}^{\circ}\right)$ at the $l$-th Monte Carlo simulation. All the numerical results provided in this section are obtained from $L=1000$ independent Monte Carlo simulations.

In the first simulation scenario, we analyze the performance of the proposed algorithm with different $N_{s}$ by setting $\sigma_{d}=$ $0.1(\mathrm{~m})$ and $\sigma_{a}=0.01(\mathrm{rad})$. The reference line in Fig. 3 (a) shows the performance of the downlink positioning proposed 
in [12] with uninformative clock bias and UE orientation, which is achieved by assistance of more than 4 single-bounce NLoS paths. We observing a number of interesting facts from Fig. 3. In all cases, having larger $N_{s}$ is beneficial, which can be explained by Lemma 1 . We can obtain the position of the UE with at least 2 RRHs, and with more than 3 RRHs, the performance of the proposed algorithm exceeds the reference line. Compared to the downlink localization, the proposed uplink localization will not be affected by the unknown antenna array orientation of the UE. To the best of our knowledge, there is not any other TDoA/FDoA/AoA-based joint velocity and position estimation algorithm in 3-D space to be included in our comparison for Fig. 3 b). Nevertheless, we can realize sub-decimeter per second level accuracy for velocity estimation with 4 or more RRHs.

In the second scenario, we consider the Cramer-Rao lower bound (CRLB) as the benchmark for accuracy of the proposed algorithm. The derivation of CRLB is given in [8], we omit details due to lack of space. We set $N_{s}=6, \sigma_{d}=40 \rho$, and $\sigma_{a}=0.1 \rho$, where $\rho$ is a scaling factor. The results shown in Fig. 4 verify the proposed TDoA/AoA positioning is more accurate than TD/AoA positioning presented in [8] due to the cancelation of clock bias, and can achieve the CRLB for small $\rho$ (noise level). Increasing the noise level results in deviating slowly from the CRLB for both position and velocity, since we ignore the nonlinear terms in $\mathbf{e}$ in derivation of the algorithm.

In the third scenario, we analyze the performance of the proposed joint estimation algorithm with different $\sigma_{d}$ and $\sigma_{a}$ by setting $N_{s}=6$. Fig. 5 infers that the proposed algorithm can be more precise with more accurate channel parameter measurements. Position is maily determined by AoAs and TDoAs, hence, the RMSE of UE position is affected by $\sigma_{d}$ and $\sigma_{a}$ at the same time. However, velocity is maily determined by FDoAs, hence, the RMSE of UE velocity is maily affected by $\sigma_{d}$ when the value of $\sigma_{d}$ is large. Actually, when $\sigma_{d}<0.1$ (m) and $\sigma_{a} \leq 0.1^{\circ}$, the estimated position can achieve subdecimeter level accuracy. Besides, when $\sigma_{d}<0.3(\mathrm{~m})$ and $\sigma_{a} \leq 1^{\circ}$, the estimated velocity can achieve sub-decimeter per second level accuracy.

\section{CONCLUSION}

This paper considered the joint position and velocity estimation in 3-D lens antenna array based mmWave CRAN. We embedded multilateration into communications and proposed a closed-form solution for the joint position and velocity estimation problem by forming the pseudo-linear matrix representation and using the WLS-based estimator. The proposed method was proven asymptotically unbiased and confirmed by simulations to achieve the CRLB and attain the sub-decimeter level accuracy.

\section{APPENDIX A}

In this Appendix, we derive the noise-free matrix representation of the joint position and velocity estimation model. Firstly, we derive $2\left(N_{s}-1\right)$ pseudo-linear TDoA and FDoA equations. Rewriting (7) as $r_{n 1}^{\circ}+r_{1}^{\circ}=r_{n}^{\circ}$ and squaring both sides yields

$$
\left(r_{n 1}^{\circ}\right)^{2}+2 r_{n 1}^{\circ} r_{1}^{\circ}=\mathbf{b}_{n}^{T} \mathbf{b}_{n}-\mathbf{b}_{1}^{T} \mathbf{b}_{1}-2\left(\mathbf{b}_{n}-\mathbf{b}_{1}\right)^{T} \mathbf{u}^{\circ} .
$$
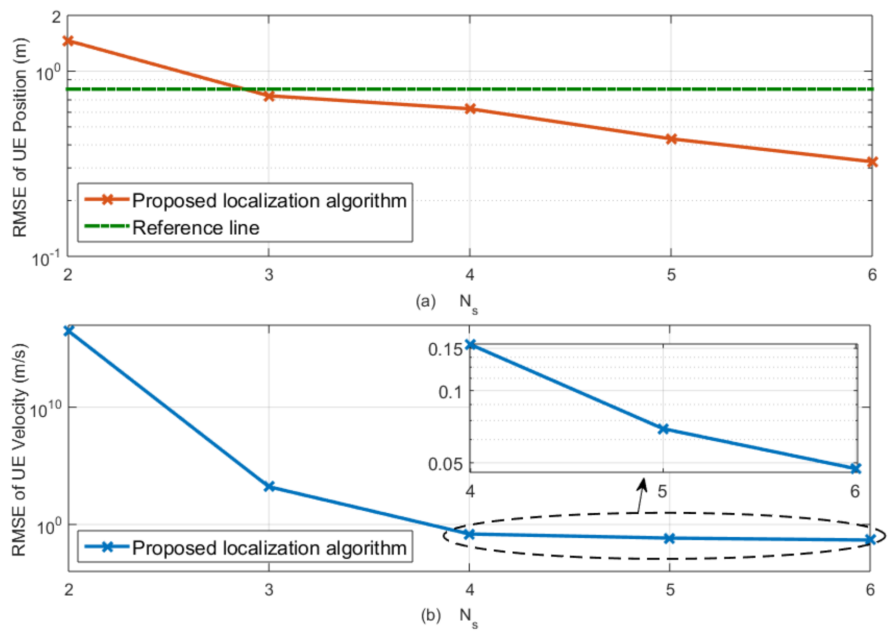

Fig. 3. RMSE as a function of $N_{s}$ for (a) position of UE and (b) velocity of UE.
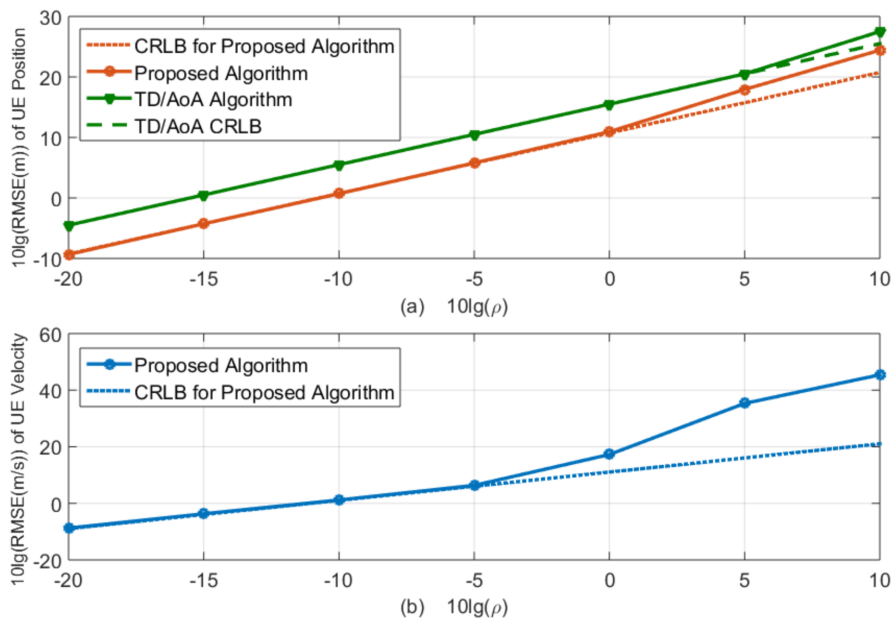

Fig. 4. Performance analysis of the presented positioning algorithm by comparison with the TD/AoA algorithm and the CRLB as a function of noise scaling factor
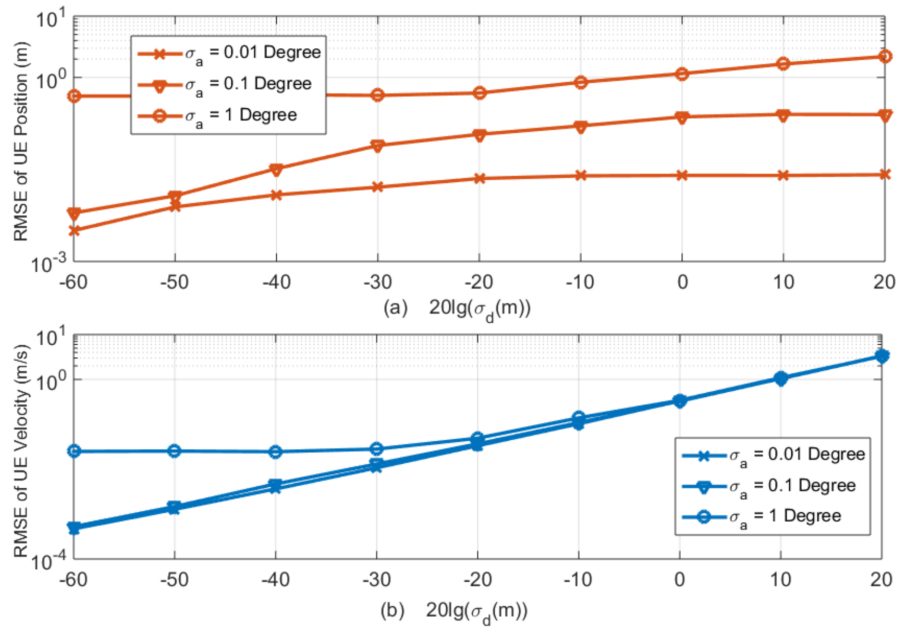

Fig. 5. RMSE as a function of $\sigma_{d}$ with given $\sigma_{a}$ for (a) position of UE and (b) velocity of UE. 
Equation (24) is pseudo-linear with respect to $\mathbf{u}^{\circ}$ and $r_{1}^{\circ}$, where $r_{1}^{\circ}$ can not be obtained directly from the channel measurement TDoA. To eliminate $r_{1}^{\circ}$, we utilize the geometrical relationship

$$
\mathbf{u}^{\circ}-\mathbf{b}_{1}=r_{1}^{\circ} \mathbf{a}_{1}^{\circ}
$$

The unit vector $\mathbf{a}_{1}^{\circ}$ possesses the following properties:

$$
\mathbf{a}_{1}^{\circ T} \mathbf{a}_{1}^{\circ}=1, \quad \dot{\mathbf{a}}_{1}^{\circ T} \mathbf{a}_{1}^{\circ}=\mathbf{a}_{1}^{\circ T} \dot{\mathbf{a}}_{1}^{\circ}=0 .
$$

Multiplying both sides of (24) by $\mathbf{a}_{1}^{\circ T} \mathbf{a}_{1}^{\circ}$ leads to

$\left(r_{n 1}^{\circ}\right)^{2}-2 r_{n 1}^{\circ} \mathbf{a}_{1}^{\circ T} \mathbf{b}_{1}-\mathbf{b}_{n}^{T} \mathbf{b}_{n}+\mathbf{b}_{1}^{T} \mathbf{b}_{1}=2\left[\left(\mathbf{b}_{1}-\mathbf{b}_{n}\right)^{T}-r_{n 1}^{\circ} \mathbf{a}_{1}^{\circ T}\right] \mathbf{u}^{\circ}$.

By taking the time derivative of 24, we have

$$
\dot{r}_{n 1}^{\circ} r_{n 1}^{\circ}+\dot{r}_{n 1}^{\circ} r_{1}^{\circ}+r_{n 1}^{\circ} \dot{r}_{1}^{\circ}=\left(\mathbf{b}_{1}-\mathbf{b}_{n}\right)^{T} \dot{\mathbf{u}}^{\circ},
$$

where $r_{1}^{\circ}$ and $\dot{r}_{1}^{\circ}$ can not be obtained directly from the channel measurements TDoA and FDoA. We will eliminate them by the geometrical relationship

$$
\dot{\mathbf{u}}^{\circ}=\dot{r}_{1}^{\circ} \mathbf{a}_{1}^{\circ}+r_{1}^{\circ} \dot{\mathbf{a}}_{1}^{\circ} .
$$

According to (26) and (29), we get

$$
\dot{r}_{n 1}^{\circ} r_{1}^{\circ}=\dot{r}_{n 1}^{\circ} \mathbf{a}_{1}^{\circ T}\left(r_{1}^{\circ} \mathbf{a}_{1}^{\circ}\right)=\dot{r}_{n 1}^{\circ} \mathbf{a}_{1}^{\circ T}\left(\mathbf{u}^{\circ}-\mathbf{b}_{1}\right),
$$

and

$$
r_{n 1}^{\circ} \dot{r}_{1}^{\circ}=r_{n 1}^{\circ} \mathbf{a}_{1}^{\circ T}\left(\dot{r}_{1}^{\circ} \mathbf{a}_{1}^{\circ}\right)=r_{n 1}^{\circ} \mathbf{a}_{1}^{\circ T}\left(\dot{\mathbf{u}}^{\circ}-r_{1}^{\circ} \dot{\mathbf{a}}_{1}^{\circ}\right)=r_{n 1}^{\circ} \mathbf{a}_{1}^{\circ T} \dot{\mathbf{u}}^{\circ} .
$$

Substituting (30) and (31) into (28), we obtain

$\dot{r}_{n 1}^{\circ} r_{n 1}^{\circ}-\dot{r}_{n 1}^{\circ} \mathbf{a}_{1}^{\circ T} \mathbf{b}_{1}=-\dot{r}_{n 1}^{\circ} \mathbf{a}_{1}^{\circ T} \mathbf{u}^{\circ}+\left[\left(\mathbf{b}_{1}-\mathbf{b}_{n}\right)^{T}-r_{n 1}^{\circ} \mathbf{a}_{1}^{\circ T}\right] \dot{\mathbf{u}}^{\circ}$.

According to 27) and 32, for $n=1, \cdots, N_{s}, 2\left(N_{s}-1\right)$ pseudo-linear TDoA and FDoA equations are obtained. Then, we derive $2 N_{s}$ AoA equations for $n=1,2, \cdots, N_{s}$, given by

$$
\mathbf{c}_{n}^{\circ T} \mathbf{b}_{n}=\mathbf{c}_{n}^{\circ T} \mathbf{u}^{\circ}, \quad \mathbf{d}_{n}^{\circ T} \mathbf{b}_{n}=\mathbf{d}_{n}^{\circ T} \mathbf{u}^{\circ} .
$$

Stacking equations (27), (32) and (33) yields $\mathbf{h}=\mathbf{G w}^{\circ}$.

\section{APPENDIX B}

We derive the WLS estimation of the unknown position and velocity vector $\mathbf{w}$. When $\mathbf{Q}$ is known, one can provide a particular choice of $\mathbf{W}$ that minimizes the variance of $\mathbf{w}$. In view of the nonlinearity of $\mathbf{e}$, it is difficult to get the weighting matrix $\mathbf{W}$ in general. According to [16], the weighting matrix that minimizes the variance of $\mathbf{w}$ is $\left(E\left\{\mathbf{e e}^{T}\right\}\right)^{-1}$, that is,

$$
\mathbf{W}=\left(E\left\{\mathbf{e e}^{T}\right\}\right)^{-1} .
$$

To find $\mathbf{W}$, we subtract (13) with (12) to obtain e, then approximate e up to the linear noise terms. Let $a_{2}=r_{1}^{\circ} r_{21}^{\circ} \dot{\phi}_{1}^{\circ} \cos ^{2} \theta_{1}^{\circ}$, $\cdots, a_{N_{s}}=r_{1}^{\circ} r_{N_{s} 1}^{\circ} \dot{\phi}_{1}^{\circ} \cos ^{2} \theta_{1}^{\circ}, b_{2}=r_{1}^{\circ} r_{21}^{\circ} \dot{\theta}_{1}^{\circ}, \cdots, b_{N_{s}}=$ $r_{1}^{\circ} r_{N_{s} 1}^{\circ} \dot{\theta}_{1}^{\circ} \cdot \dot{\phi}_{1}^{\circ}$ and $\dot{\theta}_{1}^{\circ}$ are the time derivative of 110 with $n=1$, we have

$$
\dot{\phi}_{1}^{\circ}=\frac{\mathbf{c}_{1}^{\circ T} \dot{\mathbf{u}}^{\circ}}{r_{1}^{\circ} \cos \theta_{1}^{\circ}}, \quad \dot{\theta}_{1}^{\circ}=\frac{\dot{\mathbf{u}}^{\circ T} \mathbf{d}_{1}^{\circ}}{r_{1}^{\circ}} .
$$

Then, we have

$$
\mathrm{e} \doteq \mathrm{B} \Delta \mathrm{m}
$$

where

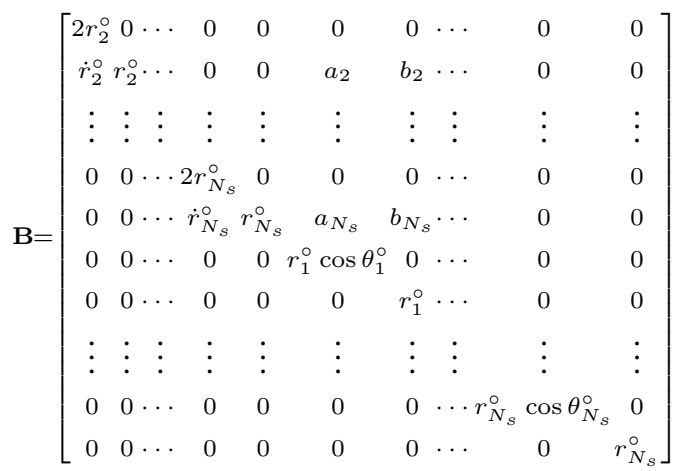

Since $E\{\Delta \mathbf{m}\}=0$ and $E\left\{\Delta \mathbf{m} \Delta \mathbf{m}^{T}\right\}=\mathbf{Q}$, the distribution of $\Delta \mathbf{m}$ implies the asymptotic distribution of $\mathbf{e}$ from $(36)$, we have $E\{\mathbf{e}\} \doteq 0$ and $\operatorname{cov}(\mathbf{e})=$ $E\left\{(\mathbf{e}-E(\mathbf{e}))(\mathbf{e}-E(\mathbf{e}))^{T}\right\} \doteq \mathbf{B Q B}^{T}$. Therefore, from (34), the weighting matrix can be easily calculated as $\mathbf{W}=$ $\left(\mathbf{B Q B}^{T}\right)^{-1}$.

\section{REFERENCES}

[1] C. X. Wang et al., "Cellular architecture and key technologies for 5G wireless communication networks," IEEE Commun. Mag., vol. 52, no. 2, pp. 122-130, Feb. 2014

[2] Y. Han, H. Zhang, S. Jin, X. Li, R. Yu and Y. Zhang, "Investigation of transmission schemes for millimeter-wave massive MU-MIMO systems," IEEE Syst. J., vol. 11, no. 1, pp. 72-83, Mar. 2017.

[3] H. Deng and A. Sayeed, "Mm-wave MIMO channel modeling and user localization using sparse beamspace signatures," in Proc. IEEE 15th Int. Workshop Signal Process. Adv. Wireless Commun. (SPAWC), Jun. 2014 pp. 130-134.

[4] J. Yang, C. K. Wen, S. Jin, and F. Gao, "Beamspace channel estimation in mmWave systems via cosparse image reconstruction technique," IEEE Trans. Commun., vol. 66, no. 10, pp. 4767-4782, Feb. 2018.

[5] R. G. Stephen and R. Zhang, "Uplink channel estimation and data transmission in millimeter-wave CRAN with lens antenna arrays," IEEE Trans. Commun., vol. 66, no. 12, pp. 6542-6555, Dec. 2018.

[6] R. D. Taranto, S. Muppirisetty, R. Raulefs, D. Slock, T. Svensson, and H. Wymeersch, "Location-aware communications for 5G networks: how location information can improve scalability, latency, and robustness of 5G," IEEE Signal Process. Mag., vol. 31, no. 6, pp. 102-112, Nov. 2014.

[7] J. Yin, Q. Wan, S. Yang, and K. C. Ho, "A simple and accurate TDoAAoA localization method using two stations," IEEE Signal Process. Lett., vol. 23, no. 1, pp. 144-148, Dec. 2016.

[8] R. Amiri, F. Behnia, and H. Zamani, "Efficient 3-D positioning using time-delay and AoA measurements in MIMO radar systems," IEEE Commun. Lett., vol. 21, no. 12, pp. 2614-2617, Aug. 2017.

[9] S. Jeong, O. Simeone, A. Haimovich, and J. Kang, "Positioning via direct localization in C-RAN systems," IET Commun., vol. 10, no. 16 , pp. 2238-2244, Jun. 2016.

[10] K. C. Ho, and W. W. Xu, "An accurate algebraic solution for moving source location using TDoA and FDoA measurements," IEEE Trans. Signal Process., vol. 52, no. 9, pp. 2453-2463, Aug. 2004.

[11] N. H. Nguyen and K. Dogancay, "Multistatic pseudolinear target motion analysis using hybrid measurements," Signal Process., vol. 130, no. C, pp. 22-36, Jan. 2017.

[12] H. Wymeersch et al., "5G mmWave downlink vehicular positioning," https://arxiv.org/abs/1901.01931v1.

[13] Y. Zeng, and R. Zhang, "Millimeter wave MIMO with lens antenna array: A new path division multiplexing paradigm," IEEE Trans. Commun., vol. 64, no. 4, pp. 1557-1571, Apr. 2016.

[14] J. Mo, P. Schniter, and R. W. Heath. Jr, "Channel Estimation in Broadband Millimeter Wave MIMO Systems with Few-Bit ADCs," IEEE Trans. Signal Process., vol. 66, no.5, pp. 1141-1154, Jan. 2018.

[15] C. N. Barati et al., "Initial access in milllimeter wave cellular systems," IEEE Trans. Wireless Commun., vol. 15, no. 12, pp. 7926-7940, Dec. 2016.

[16] S. M. Kay, Fundamentals of statistical signal processing, Estimation Theory. Englewood Cliffs, NJ, USA: Prentice-Hall, 1993. 